\title{
UTILIZAÇÃO DO JOGO LÚDICO NO ENSINO DE BIOLOGIA
}

\author{
Laís Amorim Ferreira ${ }^{1}$ \\ Luan Cerqueira de Oliveira ${ }^{2}$ \\ Mariana Aparecida Lordeiro ${ }^{3}$ \\ Elaine Gimenez Guimarães ${ }^{4}$ \\ Erika Aparecida Silva de Freitas $^{5}$ \\ Elias Terra Werner 6
}

Resumo: Os materiais didáticos são muito utilizados para o ensino de ciências e biologia, sendo assim, uma importante ferramenta para o subsidio dessas aulas. Deste modo, este trabalho teve como objetivo criar um jogo lúdico para apresentar o conteúdo de ecologia de forma mais dinâmica, como uma proposta divertida de se aprender. A confecção do jogo partiu dos conceitos abordados em sala de aula e do currículo básico comum. A atividade foi desenvolvida na Escola de Escola Estadual de Ensino Fundamental e Médio "Aristeu Aguiar", em três turmas da primeira série do ensino médio, com o auxílio dos alunos bolsistas do PIBID. Como forma de avaliação foi elaborado um texto pelos alunos relacionando os conceitos vistos durante a atividade e com a observação direta dos pibidianos. Os resultados obtidos nesse trabalho foram positivos e mostrou que a utilização do jogo lúdico como uma ferramenta útil no ensino em sala de aula, auxiliando no processo de aprendizagem.

Palavras-chave: Material didático; Ecologia; Ensino - aprendizagem.

\footnotetext{
${ }^{1}$ Graduanda em Ciências Biológicas Licenciatura/Departamento de Biologia/Centro de Ciências Exatas, Naturais e da Saúde/Universidade Federal do Espirito Santo - UFES, Brasil. E-mail: lais-guvi@hotmail.com.

2 Graduando em Ciências Biológicas Licenciatura/Departamento de Biologia/Centro de Ciências Exatas, Naturais e da Saúde/Universidade Federal do Espirito Santo - UFES, Brasil. E-mail: luaano@hotmail.com.

${ }^{3}$ Graduanda em Ciências Biológicas Licenciatura/Departamento de Biologia/Centro de Ciências Exatas, Naturais e da Saúde/Universidade Federal do Espirito Santo - UFES, Brasil. E-mail: mariana.lordeiro@gmail.com.

${ }^{4}$ Graduanda em Ciências Biológicas Licenciatura/Departamento de Biologia/Centro de Ciências Exatas, Naturais e da Saúde/Universidade Federal do Espirito Santo - UFES, Brasil. E-mail: mariana.lordeiro@gmail.com.

5 Docente/Escola Estadual de Ensino Fundamental e Médio Aristeu Aguiar, Brasil. E-mail: erikasfbr@yahoo.com.br.

${ }^{6}$ Docente/Ciências Biológicas/Departamento de Biologia/Universidade Federal do Espirito Santo, Brasil. E-mail: eliaswerner12@gmail.com.
} 